\title{
Development of an auxiliary device for ultrasound-guided aspiration of pelvic cystic masses: a simulation study
}

\author{
Baomei Yan ${ }^{1 "}$, Xiaowen Liang ${ }^{1 "}$, Jinghui Fang ${ }^{1}$, Jinsui Yu ${ }^{1}$, Zhiyi Chen ${ }^{1,2,3}$ \\ ${ }^{1}$ Department of Ultrasound Medicine, The Third Affiliated Hospital of Guangzhou Medical University, Guangzhou, China; ${ }^{2}$ Medical Imaging \\ Centre, The First Affiliated Hospital of the University of South China, Hengyang, China; ${ }^{3}$ Institute of Medical Imaging, University of South China, \\ Hengyang, China
}

"These authors contributed equally to this work.

Correspondence to: Zhiyi Chen, PhD. Medical Imaging Centre, First Affiliated Hospital of University of South China, 69 Chuanshan Road, Hengyang 421001, China. Email: winchen@vip.126.com.

Background: Pelvic cystic masses are a common gynecological condition. Ultrasound-guided aspiration is a minimally invasive surgical technique for the treatment of pelvic cystic masses. However, further developments to improve its stability and safety are wanting. This study evaluated the application and safety of a self-developed auxiliary device for pelvic cystic masses' ultrasound-guided aspiration through phantom testing.

Methods: Saline and coupling agents were used at different viscosity levels to simulate simple cysts, medium viscosity cysts (such as pelvic effusions), and ovarian, endometrial cysts. An auxiliary device consisting of a three-way valve, a negative pressure aspirator, and a pressurized infusion bag was developed. Phantom testing was performed to evaluate the application of this device in ultrasound-guided aspiration of pelvic cystic masses. The indicators, including the time of aspiration, time of injection, and the incidence of complications, were compared to cases in which ultrasound-guided aspiration was performed manually with a syringe.

Results: The incidence of complications in the auxiliary device group was significantly lower compared to the manual operation group $(\mathrm{P}<0.05)$. The ovarian cystic aspiration times and operation times were significantly shorter in the auxiliary device group compared to the traditional manual puncture group $(\mathrm{P}<0.05)$.

Conclusions: Ultrasound-guided aspiration is repeatable and minimally invasive for the treatment of pelvic cystic masses. Using the auxiliary device designed in this report resulted in shorter operation times, definite needle fixation, and fewer complications, which may allow for a more stable and safer aspiration procedure for the treatment of pelvic cystic masses.

Keywords: Ultrasound-guided aspiration; pelvic cystic mass; auxiliary device; phantom test; minimally invasive therapy

Submitted Aug 02, 2020. Accepted for publication Mar 16, 2021.

doi: 10.21037/qims-20-938

View this article at: http://dx.doi.org/10.21037/qims-20-938

\section{Introduction}

Pelvic cystic masses are a common gynecological disease with an incidence rate of approximately $7 \%$ worldwide (1). Surgery is the conventional method of treatment; however, it may cause severe trauma and pelvic adhesions. Although laparoscopy is a popular minimally invasive approach, it still requires anesthesia and can result in scarring, and these may not be acceptable for some women. Additionally, 
with laparotomy and laparoscopic surgery, there is a risk of bleeding and damage to the ovarian artery, leading to adverse effects on ovarian function (2). Ultrasound-guided aspiration of pelvic cystic masses is another minimally invasive technique that has been widely used in recent years due to its ease of scheduling, reduced costs, and rapid recovery $(3,4)$. Simple cysts $(5)$, pelvic abscesses $(6)$, hydrosalpinx in cases of infertility (7), and ovarian, endometrial cysts (8) are the most common indications. Unfortunately, ultrasound-guided aspiration can be complicated by the risk of unwanted needle displacement and damage to the cyst wall. If patients are given ethanol sclerotherapy following aspiration, such complications may result in hardening agent leakage into the pelvis. Also, the time-consuming and mechanized operation can be a burden to physicians (9). These problems have limited the clinical application of ultrasound-guided aspiration in the treatment of pelvic cystic masses. In recent decades, minimally invasive therapy in pelvic diseases treatment has been regarded as an efficient diagnostic and alternative therapeutic procedure $(10,11)$. However, to the best of our knowledge, few studies have examined the use of auxiliary devices for ultrasoundguided aspiration in the treatment of pelvic cystic masses.

The viscosity of cystic masses is a major consideration in using auxiliary devices during the aspiration procedure. Much research has been conducted examining the relationship between the cyst content's viscosity and the successful treatment of cysts (12). Depending on the cystic fluid contents, different types of cysts (such as simple ovarian cysts, pelvic abscess, and parcel effusions) have different viscosity, which may impact the aspiration procedure. In this report, the best fit viscosity of the cyst model was investigated. For example, ovarian, endometrial cysts are the most common pelvic cystic masses and have a high viscosity. Since this cyst's main content is hematocele, a concentration similar to blood viscosity was selected as the cyst model for ovarian, endometrial cysts.

A convenience auxiliary device was developed for ultrasound-guided aspiration in treating pelvic cystic masses. By using the device, the influence of artificial factors was reduced. The operation time was also shortened, which reduced the working intensity of doctors. The auxiliary device was combined with a negative pressure aspirator, a pressurized infusion bag, and a three-way valve. The pressurized infusion bag is a convenient and safe necessity for clinical emergency treatment.

Moreover, some studies advocate the application of pressurized infusion bags, except in cases of transfusions.
For example, Dewaele et al. used a pressurized infusion bag to enforce rinsing and then increased the head model's pressure to simulated high intracranial pressure (13). This device is simple and relatively easy to popularize. Furthermore, combined with the viscosity test, simulation tests were applied to compare our device's advantages with the traditional manual puncture technique.

\section{Methods}

\section{Equipment and supplies}

The ultrasound-guided aspiration auxiliary device consisted of a YB-DX23D negative pressure aspirator (SMAF, Shanghai, China), a pressurized infusion bag with a pressure range of $0-300 \mathrm{mmHg}$, and a three-way valve. Voluson S8 (GE Healthcare, Wauwatosa, USA) with a transducer of RIC5-9W-RS (3.9-8.0 MHz) was used for imaging. Other supplies included physiological saline, coupling agent, syringes $(5 \mathrm{~mL}$ and $30 \mathrm{~mL}$ ), disposable infusion sets, condoms, rubber balloons, dressing boxes, follicular puncture needles $(17 \mathrm{G})$, and infusion stands $(180 \mathrm{~cm})$. A $5 \mathrm{~mL}$ syringe was connected to the hose connector of the negative pressure aspirator. A disposable infusion set was connected to the physiological saline bag $(100 \mathrm{~mL})$ used for flushing. Finally, the three-way valve was used to form the device by connecting the two parts mentioned above.

\section{Modeling}

An empty box filled with coupling agent was regarded as the pelvic cavity. The coupling agent, at different concentrations, was used with physiological saline to simulate cysts of different viscosities. The blood viscosity of $5 \mathrm{~mm}^{2} / \mathrm{s}$ at $25{ }^{\circ} \mathrm{C}$ was selected as the highest viscosity, and this concentration was halved to generate the medium viscosity model. This resulted in coupling agent concentrations of $2.33 \%$ and $4.65 \%$. After 24 hours, $50 \mathrm{~mL}$ of physiological saline, $2.33 \%$ coupling agent, and $4.65 \%$ coupling agent was filled into separate balloons. There were 30 balloons in each group, 15 in the experimental sub-group and 15 in the control sub-group. The equipment, phantom model, and cyst models are shown in Figure 1.

\section{Velocity test}

Pressure, ranging from $0.02-0.095 \mathrm{MPa}$, was used to aspirate physiological saline, the $2.33 \%$ coupling agent 

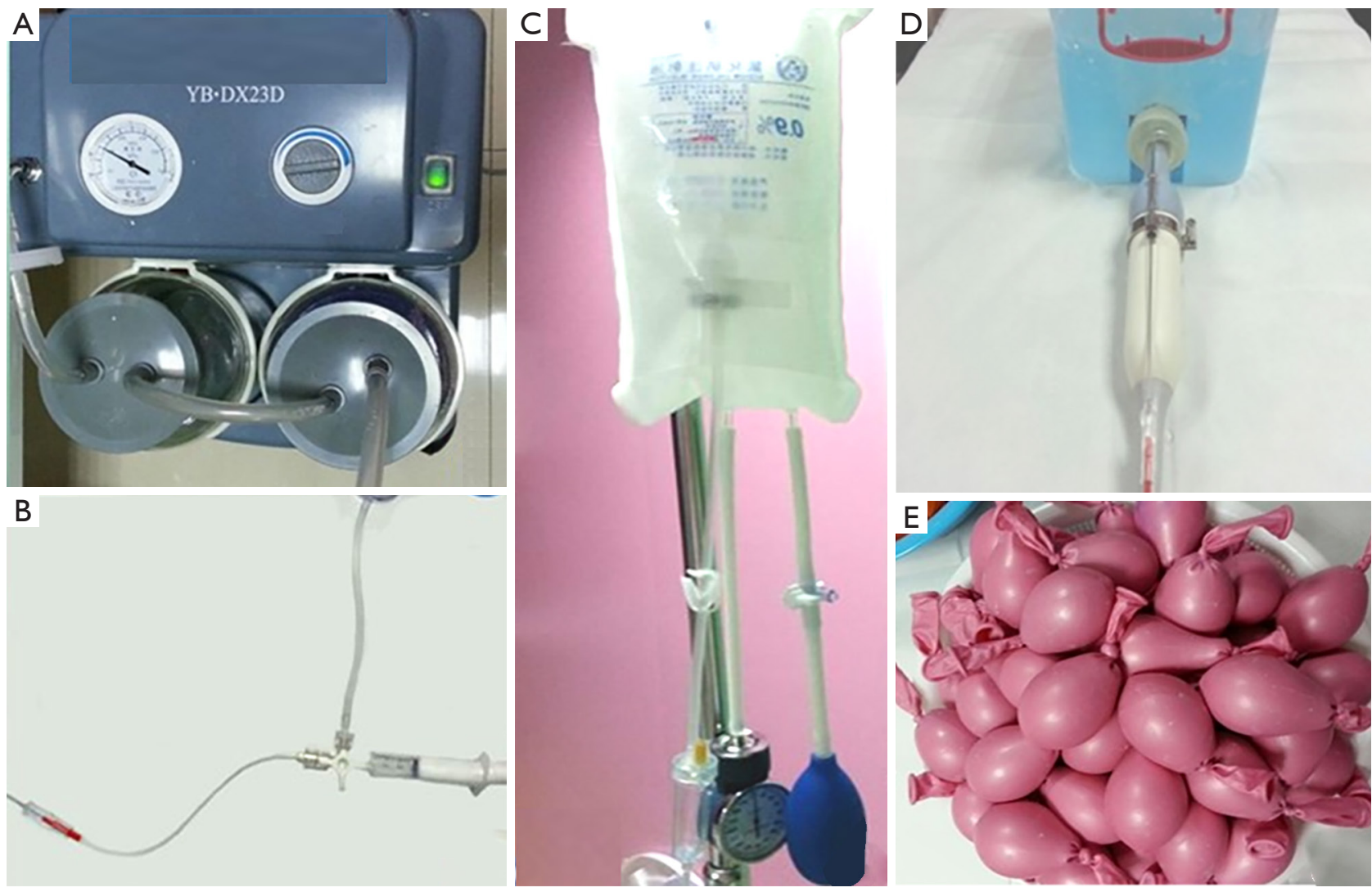

Figure 1 The auxiliary device for ultrasound-guided aspiration in the treatment of pelvic cystic masses. (A) The negative pressure aspirator. (B) The three-way valve. (C) The pressurized infusion bag and physiological saline bag. (D) The phantom consisted of the puncture groove, puncture frame, and transvaginal probe. (E) The cyst models with different viscosities.

solution, and the $4.65 \%$ coupling agent solution. The suction times were recorded. Meanwhile, physiological saline was infused under pressure $(20-300 \mathrm{mmHg})$, and the infusion times were recorded. The following calculation formula was used: Flow rate $=$ volume $/$ time $(\mathrm{mL} / \mathrm{s})$.

\section{Phantom test}

Contrast tests were applied to evaluate the application of the auxiliary device. In the experimental group, the auxiliary device was used, and in the control group, manual aspiration was performed using a syringe. The experimental and control groups were carried out by three people, the probe holder, the operator, and the assistant. The job of the probe holders was to support the probe.

In the control group, the pelvic cyst models were placed in the assistant's puncture groove, and the operator quickly punctured the pelvic cyst model under the guidance of ultrasound. The needle was connected to a $30 \mathrm{~mL}$ syringe to aspirate the fluid inside the cyst model; then, the cyst wall was washed with $60 \mathrm{~mL}$ physiological saline. The assistant was responsible for the transmission of physiological saline.

In the experimental group, the pelvic cyst models were also placed in the assistant's puncture groove, and $60 \mathrm{~mL}$ physiological saline was injected into the bag. The assistant adjusted the pressure of the infusion pressure bag and the negative pressure attractor. The operator then connected the puncture needle to the auxiliary device and quickly punctured the pelvic cyst model. The cyst fluid was aspirated with a negative pressure of $0.08 \mathrm{MPa}$. Due to the cysts' deformation during aspiration, the puncture needle's position was adjusted appropriately to avoid the destruction of the cyst wall. When there was about $5-10 \mathrm{~mL}$ cyst fluid remaining, the operator adjusted the three-way valve and infused the cyst model with physiological saline. The infusion pressure was controlled at 140-160 mmHg. The above suction was repeated until the 

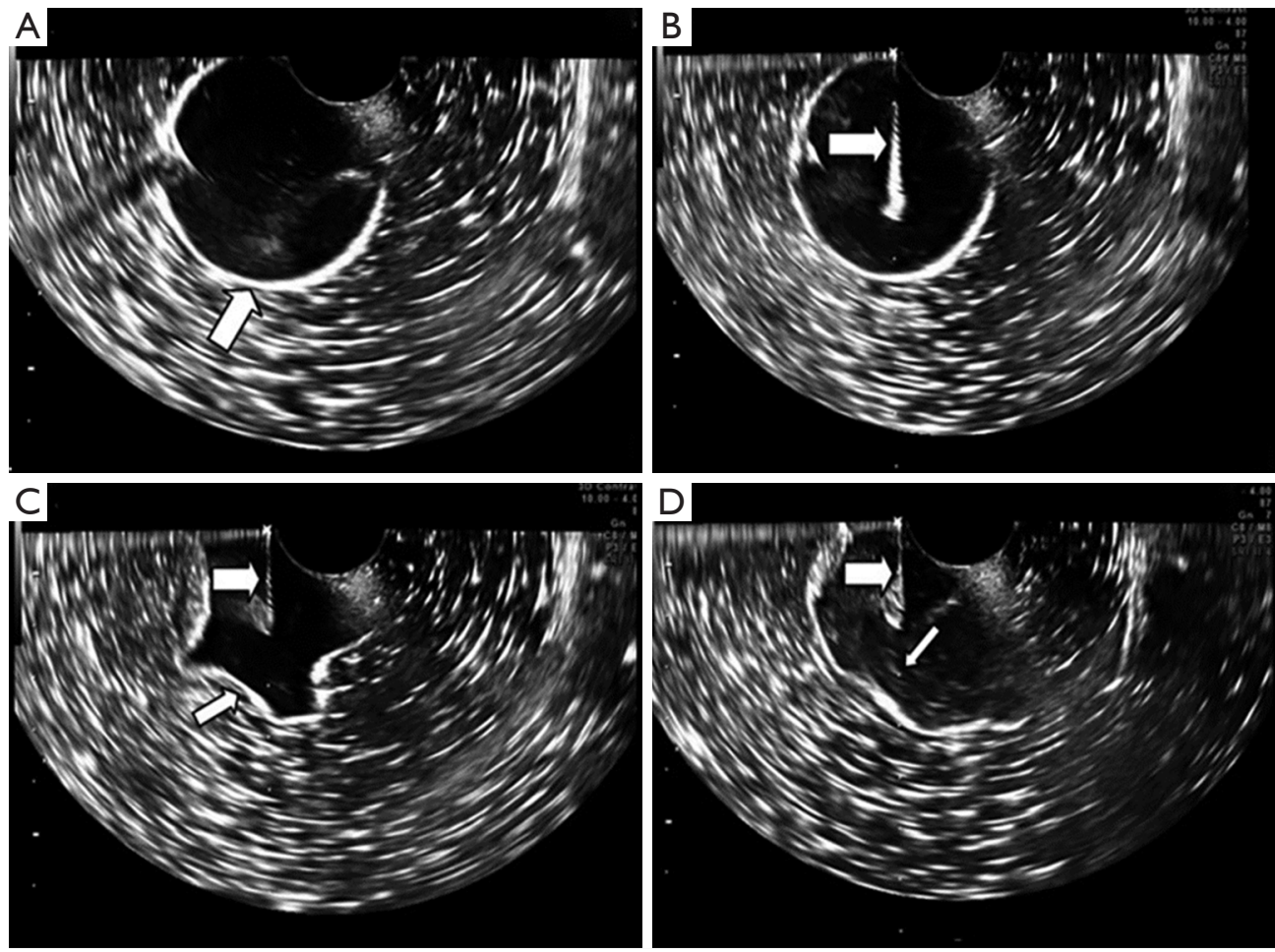

Figure 2 The procedure of ultrasound-guided aspiration using the auxiliary device. (A) The pelvic cyst model before operation (arrow shows the cyst wall). (B) Puncture needle was inserted into the cyst model (arrow shows the puncture needle). (C) Cyst fluid was aspirated. (the thick arrow shows the needle point, and the thin arrow shows the collapsed cyst wall). (D) The infusion pressure was applied to flush the cyst model (Thick arrow shows the needle point, and the thin arrow shows a bubble inside the cyst model).

physiological saline was depleted. The time of aspiration, time of fluid injection, the washing time, and the operation time were recorded. The steps involved in the whole procedure are shown in Figure 2.

\section{Statistical analysis}

Flow rate data were processed with the PASW Statistics 18 software using scatter plots. Contrast test results were analyzed using the SPSS17.0 statistical software. Oneway analysis of variance, Kruskal-Wallis, Mann-Whitney, independent sample t-tests, and chi-square tests was used to analyze the significance of the differences, and $\mathrm{P}<0.05$ was considered statistically significant.

\section{Results}

\section{Experiment conditions}

In these experiments, the cyst model's viscosity with a concentration of $4.65 \%$ was relatively close to the average viscosity of blood $\left(5.00 \mathrm{~mm}^{2} / \mathrm{s}\right)$ at $25{ }^{\circ} \mathrm{C}$ (Table 1). Also, the closer the pressure was to the limit negative pressure, the smaller the increase in flow rate. According to the three cyst models' aspiration time and flow rate curve with different viscosities, the appropriate suction pressure range was approximately $0.05-0.08 \mathrm{MPa}$.

\section{Aspiration velocity}

In the presence of the auxiliary device, increasing fluid viscosity in the cyst models resulted in increased aspiration time and decreased average flow rate (Table 2). In all 3 cyst models, the rate of decrease in aspiration time and the rate of increased flow velocity were significantly hampered at the threshold values of $0.055 \mathrm{MPa}, 0.050 \mathrm{MPa}$, and $0.075 \mathrm{MPa}$ (Figure 3).

\section{Fluid injection rate}

When the pressure was raised to $140 \mathrm{mmHg}$ and above, the 
Table 1 The viscosities of the coupling agents $\left(25^{\circ} \mathrm{C}\right)$

\begin{tabular}{lcc}
\hline $\begin{array}{l}\text { Concentration of the } \\
\text { cyst model }(\mathrm{g} / \mathrm{mL})\end{array}$ & Average time $(\mathrm{s})$ & Viscosities $\left(\mathrm{mm}^{2} / \mathrm{s}\right)$ \\
\hline 0.0588 & 3,132 & 7.83 \\
0.0571 & 2,892 & 7.23 \\
0.0526 & 2,441 & 6.10 \\
0.0500 & 2,263 & 5.66 \\
0.0465 & 2,036 & 5.09 \\
\hline
\end{tabular}

Table 2 Aspiration velocities of the auxiliary device under various conditions

\begin{tabular}{lcc}
\hline Cyst content & Average time $(\mathrm{s})$ & $\begin{array}{c}\text { Average flow } \\
\text { rate }(\mathrm{mL} / \mathrm{s})\end{array}$ \\
\hline Physiological saline & $32.11 \pm 11.65$ & $1.70 \pm 0.46$ \\
$2.33 \%$ coupling agent & $34.47 \pm 16.42$ & $1.67 \pm 0.54$ \\
$4.65 \%$ coupling agent & $71.13 \pm 42.82$ & $0.92 \pm 0.41$ \\
\hline
\end{tabular}
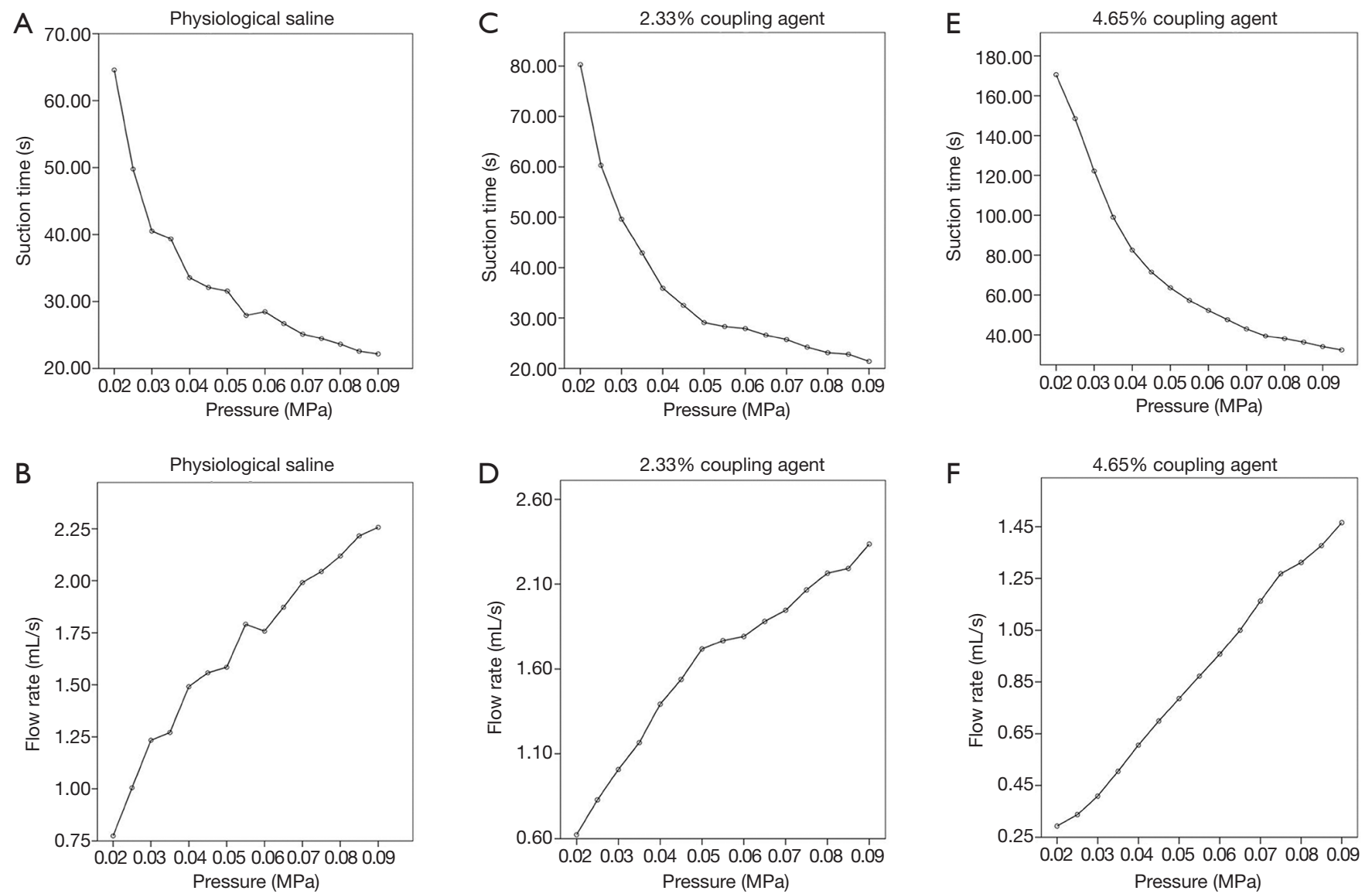

Figure 3 The dynamic changes in aspiration time and flow rate with pressure. (A) The change in aspiration time with pressure in the physiological saline model. (B) The change of flow rate with pressure in the physiological saline model. (C) The change of aspiration time with pressure in the $2.33 \%$ coupling agent model. (D) The change of flow rate with pressure in the $2.33 \%$ coupling agent model. (E) The change of aspiration time with pressure in the $4.65 \%$ coupling agent model. (F) The change of flow rate with pressure in the $4.65 \%$ coupling agent model. 

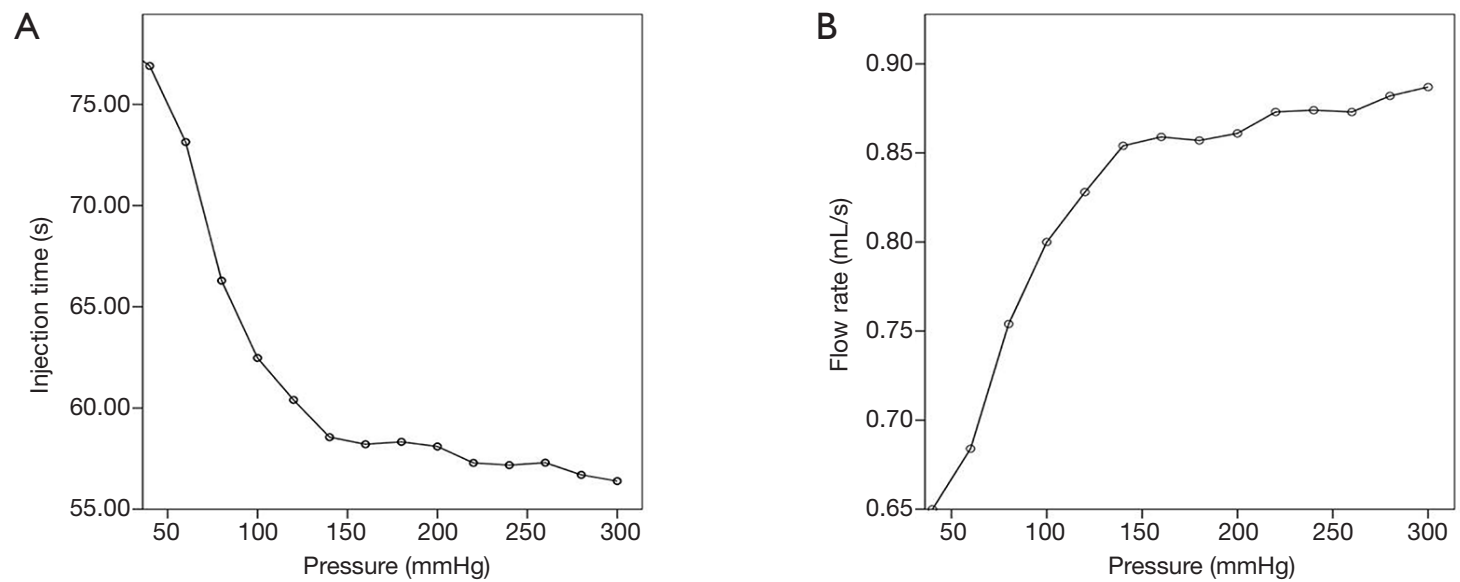

Figure 4 The curve of injection time and injection flow rate with pressure. (A) The change of injection time with pressure. (B) The change of injection flow rate with pressure.

Table 3 Complications of manual aspiration and auxiliary device

\begin{tabular}{lccc}
\hline Indicators & Manual aspiration $(\mathrm{n}=45)$ & Auxiliary device $(\mathrm{n}=45)$ & $\mathrm{P}$ value \\
\hline Separation [n (\%)] & $4(8.9)$ & $0(0)$ & 0.125 \\
Needle stuck to the cyst wall [n (\%)] & $5(11.1)$ & $2(4.4)$ & 0.431 \\
Unstable fixation [n (\%)] & $1(2.2)$ & $0(0)$ & 1.000 \\
Total [n (\%)] & $10(22.2)$ & $2(4.4)$ & $0.013^{\star}$ \\
\hline
\end{tabular}

${ }^{*} \mathrm{P}<0.05$.

rate of decrease in the injection time and the rate of increase in injection velocity were gradually diminished (Figure 4).

\section{The incidence rate of complications}

The incidence rate of complications during the experimental procedure was assessed. During the aspiration, the control group separation of the syringe and the puncture needle occurred 4 times, and the needle stuck to the cyst wall on 5 occasions. During the injection, unstable fixation of the syringe and the puncture needle occurred once.

In the experimental group, both the aspiration and the injection procedures were performed successfully, except the needle was stuck to the cyst wall on 2 occasions. The difference in the incidence rate of total complications between the two treatment groups was statistically significant $(\mathrm{P}<0.05$; Table 3).

\section{The impact of different viscosities}

In both the experimental and control groups, the shortest aspiration time was observed in the physiological saline model and longest in the $4.65 \%$ coupling agent model $(\mathrm{P}<0.05)$. There were no significant differences between the two groups in terms of washing time $(\mathrm{P}>0.05)$. Tables 4,5 lists the comparison of both groups with the different viscosity models.

\section{Application performance of the auxiliary device}

Regardless of the cyst content's viscosity, the aspiration times and operation times were shorter in the experimental group compared with the manual aspiration group $(\mathrm{P}<0.05)$. Washing time of the $2.33 \%$ coupling agent was shorter in the experimental group than the control group, while there was no significant difference between the two groups in the physiological saline model and the $4.64 \%$ coupling agent model $(\mathrm{P}>0.05$; Tables 6-8).

\section{Discussion}

Interdisciplinary integration has solved many problems 
Table 4 Operation time of the different viscosity models using the auxiliary device

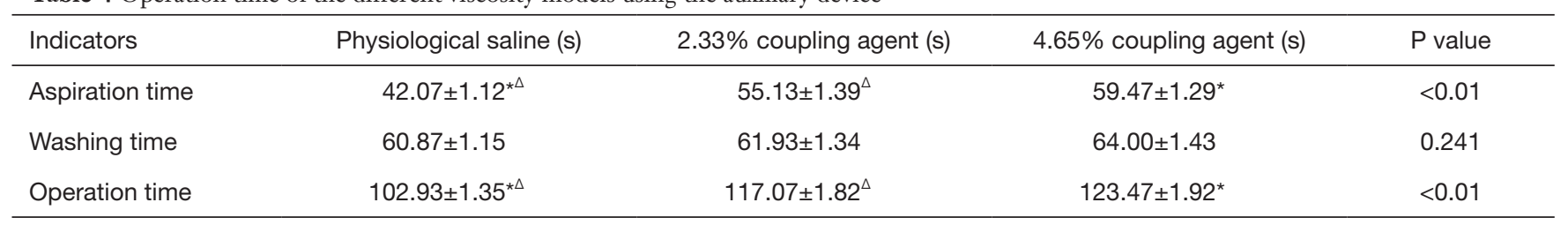

${ }^{*}$ Compared with $2.33 \%$ coupling agent, $\mathrm{P}<0.05 .{ }^{\Delta}$ Compared with $4.64 \%$ coupling agent, $\mathrm{P}<0.05$.

Table 5 Operation time of the different viscosity models using manual aspiration with a syringe

\begin{tabular}{|c|c|c|c|c|}
\hline Indicators & Physiological saline (s) & $2.33 \%$ coupling agent (s) & $4.65 \%$ coupling agent (s) & $P$ value \\
\hline Aspiration time ${ }^{a}$ & $93^{\star \Delta}$ & $105^{\Delta}$ & $111^{*}$ & $<0.01$ \\
\hline Washing time ${ }^{b}$ & $64.40 \pm 2.07$ & $65.87 \pm 1.24$ & $67.60 \pm 1.37$ & 0.377 \\
\hline Operation time ${ }^{b}$ & $154.27 \pm 2.71^{\star \Delta}$ & $170.67 \pm 2.25^{\Delta}$ & $178.67 \pm 1.65^{\star}$ & $<0.01$ \\
\hline
\end{tabular}

${ }^{a}$ The data did not conform to a normal distribution $(\mathrm{P}<0.05)$, and the data are presented as mean $(\mathrm{M})$. ${ }^{\mathrm{b}}$ The data conformed to a normal distribution $(P>0.05)$, and the variance was homogeneous. The data are presented as mean \pm standard error $(\chi \pm S E)$. ${ }^{*} \mathrm{Compared}$ with $2.33 \%$ coupling agent, $\mathrm{P}<0.05$. ${ }^{\triangle}$ Compared with $4.64 \%$ coupling agent, $\mathrm{P}<0.05$.

Table 6 Application performance of manual aspiration and the auxiliary device in the physiological saline model

\begin{tabular}{lccc}
\hline Indicators & Manual aspiration & Auxiliary device & P value \\
\hline Aspiration time $^{\mathrm{a}}$ & 93 & 42 & $<0.01$ \\
Washing time $^{\mathrm{b}}$ & $64.40 \pm 2.07$ & $60.87 \pm 1.15$ & 0.151 \\
Operation time $^{\mathrm{b}}$ & $154.27 \pm 2.71$ & $102.93 \pm 1.35$ & $<0.01$ \\
\hline
\end{tabular}

${ }^{a}$ The data did not conform to a normal distribution $(\mathrm{P}<0.05)$, and the data are presented as mean $(\mathrm{M}){ }^{\mathrm{b}}$ The data conformed to a normal distribution $(P>0.05)$, and the variance was homogeneous. The data are presented as mean \pm standard error.

Table 7 Application performance of manual aspiration and the auxiliary device in the $2.33 \%$ coupling agent model

\begin{tabular}{lccc}
\hline Indicators & Manual aspiration & Auxiliary device & P value \\
\hline Aspiration time (s) & $104.80 \pm 2.18$ & $55.13 \pm 1.39$ & $<0.01$ \\
Washing time (s) & $65.87 \pm 1.24$ & $61.93 \pm 1.34$ & 0.040 \\
Operation time (s) & $170.67 \pm 2.25$ & $117.07 \pm 1.82$ & $<0.01$ \\
\hline
\end{tabular}

Table 8 Application performance of manual aspiration and the auxiliary device in the $4.65 \%$ coupling agent model

\begin{tabular}{lccc}
\hline Indicators & Manual aspiration & Auxiliary device & P value \\
\hline Aspiration time (s) & $111.07 \pm 1.98$ & $59.47 \pm 1.29$ & $<0.01$ \\
Washing time (s) & $67.60 \pm 1.37$ & $64.00 \pm 1.43$ & 0.079 \\
Operation time (s) & $178.67 \pm 1.65$ & $123.47 \pm 1.92$ & $<0.01$ \\
\hline
\end{tabular}


in the medical field (14). In this research, a convenience auxiliary device was developed for ultrasound-guided aspiration to treat pelvic cystic masses. The performance of this device was verified through phantom tests. The experimental results demonstrated that the auxiliary device had the features of a shorter operation time, definite needle fixation, and fewer complications. This simple device may eliminate many problems associated with manual aspirations during ultrasound-guided aspiration.

Clinically, negative pressure suction is applied during trans-ultrasound-guided puncture treatment of ovarian cysts, and the accepted pressure range is still controversial. The pressure depends on the viscosity of the capsule fluid. For cystic lesions with high viscosity, high negative pressure is generally used for suction. However, Suys E's research results in other parts show that when the pressure is too high, the drainage tube will be blocked and the flow will drop to zero (15). Although pelvic cysts' fluid does not generally block the aspiration needle, it should be noted that with low viscosity, the needle tubing may easily become stuck to the cystic wall. This current investigation showed that the suction speed of the negative pressure decreased with increasing concentration. According to the suction time and velocity scatter curve, the appropriate suction pressure range was about $0.05-0.08 \mathrm{Mpa}$.

In the clinical application of the pressurized infusion bag for pelvic cyst, the infusion rate is related to the pressure of the pressurized infusion bag, the volume of liquid in the saline bag, the specification of the infusion tube, the height of the infusion bag, and the size and depth of the lesion. In this study, the scatter plot examining the infusion time and the flow rate of the fluid under different pressure of the pressurized infusion bag showed that pressure did not have a directly proportional relationship with flow rate. Instead, increasing pressure progressively reduced the flow rate increase, especially after a pressure point of $140 \mathrm{mmHg}$. Therefore, we suggest that the appropriate infusion pressure range for the pressurized infusion bag is between 140-160 mmHg. In the same volume of liquid, the smaller the syringe's volume, the more times suction is required. Clinically, a $20-30 \mathrm{~mL}$ syringe is typically used for cyst fluid aspiration. Since the cyst model volume was $50 \mathrm{~mL}$, the number of aspiration attempts, the number of times the needle is injected, and the number of times the syringe is connected to the puncture needle is much less for a $30 \mathrm{~mL}$ syringe compared to a $20 \mathrm{~mL}$ syringe. This will consequently reduce the operation time and the probability of puncture complications.
When using the auxiliary device, during the process of aspiration and washing, the operator only needs to adjust the puncture needle's position by ultrasound without force. This allows adjustments to the puncture needle's position in a timely and accurate manner to prevent the needle from sticking to the cyst wall or damaging the cyst wall. This reduces the risk of cyst rupture or leakage of the cyst fluid. Also, the operator can adjust the position of the puncture needle with one hand and support the probe with the other hand to temporarily replace the fixation of the ultrasonic probe to alleviate the fatigue of the probe holder and make the position of the puncture needle more stable. However, in the control group, the operator needs to fix the syringe's connection and the puncture needle with one hand while using the other hand for the suction, infusion, and flushing. In the process of fluid extraction, the hard suction of the capsule fluid may distract the operator's attention. Moreover, due to the need to repeatedly connect and disconnect the needle and the syringe, using the syringe for aspiration may lead to more errors.

In this study, the fluid viscosity of the cyst model impacted both treatment modes. The higher the fluid viscosity, the longer the aspiration time and the total operation time. There was no significant difference in the flushing time for different fluid viscosities. The higher the fluid viscosity, the greater the resistance during the suction process. In this study, the suction time was significantly shorter for the physiological saline model and longer for the $4.65 \%$ coupling agent model than the $2.33 \%$ coupling agent model. However, no significant differences were detected in the flushing time as the same volume of physiological saline was used for flushing in all three models. In the experimental group, the infusion pressure bag was used, and this required fewer procedural steps, but the process was slower.

In contrast, the syringe method was used in the control group, and this required more procedural steps, but the process was faster. Also, at the same fluid viscosity of the cyst model, the auxiliary device groups' aspiration and total times were significantly shorter than those observed in the manual syringe group. The longer aspiration time experienced in the syringe group above is mainly due to the repetition of multi-procedural steps by human labor compared to the auxiliary device. The experimental group's operation time was shorter than that of the control group, suggesting that the auxiliary device method be more efficient in clinical practice.

Overall, these findings suggested that the auxiliary device is a safe, stable, and effective alternative treatment option 
for pelvic cystic masses. García-Tejedor et al. (10) reported that the major and minor complications of interventional surgery included severe inflammatory diseases and hemoperitoneum. Using the auxiliary device can effectively reduce the incidences of such complications and shorten the operation time. This interventional treatment auxiliary device for pelvic cystic mass meets the basic design criteria of medical devices through experimental verification, namely safety, efficacy, stability, and flexibility. Compared with manual aspiration using a syringe, the use of this auxiliary device reduces the need for repeated multiprocedural steps and does not require force, all of which make this auxiliary device an effective option in clinical practice.

There are still some limitations in this study. First, the pressurized infusion bag used in this study is not selfadapting, and thus, the fluid infusion rate and volume must be carefully monitored in case of excessive infusion. Also, the pressure produced by the pressurized infusion bag was not as strong as that produced by a syringe, which resulted in less effective flushing. Furthermore, other factors such as cyst wall thickness and intracapsular septation have not been examined. Future research addressing these issues is warranted.

\section{Conclusions}

Ultrasound-guided aspiration is repeatable and minimally invasive for the treatment of pelvic cystic masses. However, refinements are necessary to improve stability and reduce the incidences of complications. The study identified the appropriate suction pressure range to be approximately 0.05-0.08 Mpa. An auxiliary device was developed with the features of a shorter operation time, definite needle fixation, and fewer complications, all of which may lead to more stable and safer aspiration in patients with pelvic cysts. In addition to its application in clinical practice, this device and the models developed in this report can be used as a teaching tool for clinical skills training. Further clinical trials should be conducted in the future to study the influence of other factors on puncture stability and to verify the clinical reliability and practicability of auxiliary equipment. With further improvements, this auxiliary device has great clinical and teaching potential.

\section{Acknowledgments}

Funding: This work was supported in part by the Major
Research Projects of Universities in Guangdong Province (No. 2019KZDZX1032), the Scientific and Technological Livelihood Projects of Liwan District (No. 201904003), and the Youth Foundation of Scientific Research of the Third Affiliated Hospital of Guangzhou Medical University (No. 2018Q18).

\section{Footnote}

Conflicts of Interest: All authors have completed the ICMJE uniform disclosure form (available at http://dx.doi. org/10.21037/qims-20-938). Dr. ZC serves as an unpaid editorial board member of Quantitative Imaging in Medicine and Surgery. All other authors have no conflicts of interest to declare.

Ethical Statement: This study is based on a simulation model of an auxiliary device for pelvic cystic mass interventional treatment in vitro. This study did not use humans or animals as research subjects. No human or animal experiments were conducted, and no ethical issues were involved.

Open Access Statement: This is an Open Access article distributed in accordance with the Creative Commons Attribution-NonCommercial-NoDerivs 4.0 International License (CC BY-NC-ND 4.0), which permits the noncommercial replication and distribution of the article with the strict proviso that no changes or edits are made and the original work is properly cited (including links to both the formal publication through the relevant DOI and the license). See: https://creativecommons.org/licenses/by-nc-nd/4.0/.

\section{References}

1. Farghaly SA. Current diagnosis and management of ovarian cysts. Clin Exp Obstet Gynecol 2014;41:609-12.

2. Ata B, Turkgeldi E, Seyhan A, Urman B. Effect of hemostatic method on ovarian reserve following laparoscopic endometrioma excision; comparison of suture, hemostatic sealant, and bipolar dessication. A systematic review and meta-analysis. J Minim Invasive Gynecol 2015;22:363-72.

3. Ahmed HU, El-Shater Bosaily A, Brown LC, Gabe R, Kaplan R, Parmar MK, Collaco-Moraes Y, Ward K, Hindley RG, Freeman A, Kirkham AP, Oldroyd R, Parker C, Emberton M; PROMIS study group. Diagnostic accuracy of multi-parametric MRI and TRUS biopsy in prostate cancer (PROMIS): a paired validating 
confirmatory study. Lancet 2017;389:815-22.

4. Dietrich CF. EFSUMB guidelines 2015 on interventional ultrasound. Med Ultrason 2015;17:521-7.

5. Bannon LC, Hayes KG, Porter KB. Percutaneous Drainage of a Rapidly Enlarging Simple Ovarian Cyst in the Third Trimester. Mil Med 2015;180:e1118-20.

6. Zhou Y, Jiang H, Zhang WX, Ni F, Wang XM, Song XM. Ultrasound-guided aspiration of hydrosalpinx occurring during controlled ovarian hyperstimulation could improve clinical outcome of in vitro fertilization-embryo transfer. J Obstet Gynaecol Res 2016;42:960-5.

7. Akıncı D, Ergun O, Topel Ç, Çiftçi T, Akhan O. Pelvic abscess drainage: outcome with factors affecting the clinical success. Diagn Interv Radiol 2018;24:146-52.

8. Wu X, Xu Y. Gestrinone combined with ultrasound-guided aspiration and ethanol injection for treatment of chocolate cyst of ovary. J Obstet Gynaecol Res 2015;41:712-6.

9. Castellarnau Visus M, Ponce Sebastia J, Carreras Collado R, Cayuela Font E, Garcia Tejedor A. Preliminary results: ethanol sclerotherapy after ultrasound-guided fine needle aspiration without anesthesia in the management of simple ovarian cysts. J Minim Invasive Gynecol 2015;22:475-82.

Cite this article as: Yan B, Liang X, Fang J, Yu J, Chen Z. Development of an auxiliary device for ultrasound-guided aspiration of pelvic cystic masses: a simulation study. Quant Imaging Med Surg 2021;11(7):3165-3174. doi: 10.21037/qims-20938
10. García-Tejedor A, Castellarnau M, Burdio F, Fernández E, Martí D, Pla MJ, Ponce J. Ultrasound-guided aspiration of adnexal cysts with a low risk of malignancy: is it a recommendable option? J Ultrasound Med 2015;34:985-91.

11. Kostrzewa M, Zając A, Wilczyński JR, Stachowiak G. Retrospective analysis of transvaginal ultrasound-guided aspiration of simple ovarian cysts. Adv Clin Exp Med 2019;28:1531-5.

12. Clarke L, Edwards A, Graham E. Acoustic streaming: an in vitro study. Ultrasound Med Biol 2004;30:559-62.

13. Vincent CJ, Li Y, Blandford A. Integration of human factors and ergonomics during medical device design and development: it's all about communication. Appl Ergon 2014;45:413-9.

14. Phei Er Saw, Shanping Jiang. The Significance of Interdisciplinary Integration in Academic Research and Application. BIO Integration 2020;1:2-5.

15. Suys E, Nieboer K, Stiers W, De Regt J, Huyghens L, Spapen H. Intermittent subglottic secretion drainage may cause tracheal damage in patients with few oropharyngeal secretions. Intensive Crit Care Nurs 2013;29:317-20. 